\title{
ELECTROMAGNETIC POLLUTION OF NEAR-EARTH SPACE BY POWER LINE EMISSION
}

\author{
V.A. Pilipenko \\ Space Research Institute RAS, \\ Moscow, Russia, pilipenko_va@mail.ru \\ Institute of Physics of the Earth RAS, \\ Moscow, Russia \\ E.N. Fedorov \\ Institute of Physics of the Earth RAS, \\ Moscow, Russia, enfedorov1@yandex.ru
}

\author{
N.G. Mazur \\ Institute of Physics of the Earth RAS, \\ Moscow,Russia,ngmazur@mail.ru \\ S.I. Klimov \\ Space Research Institute RAS, \\ Moscow,Russia,sklimov@cosmos.ru
}

\begin{abstract}
We present an overview, based on satellite observations at low Earth orbits, on electromagnetic radiation from ground power transmission lines at an industrial frequency 50-60 Hz. Particular attention has been given to Chibis-M and DEMETER satellite observations. The electric $40-\mathrm{cm}$ antenna of the microsatellite often recorded $50-60 \mathrm{~Hz}$ radiation (known as Power Line Emission (PLE)) when it flew over industrialized areas of the planet. The PLE spectral amplitude varied from 1.2 to $18(\mu \mathrm{V} / \mathrm{m}) / \mathrm{Hz}^{0.5}$, which corresponds to the electric field amplitude $E \sim 1 \mu \mathrm{V} / \mathrm{m}$. We report results of numerical calculations of the electromagnetic response of the atmosphere and ionosphere to a largescale surface emitter at a frequency of $50 \mathrm{~Hz}$. According to simulation results, PLE with an intensity of $\sim 1 \mu \mathrm{V} / \mathrm{m}$ observed on satellites in the nightside ionosphere at midlatitudes can be excited by an unbalanced current 8 $10 \mathrm{~A}$ in a power transmission line above the earth's crust
\end{abstract}

with conductivity of $10^{-3} \mathrm{~S} / \mathrm{m}$. At middle and low latitudes with an inclined geomagnetic field, the maximum response in the upper ionosphere to the transmission line radiation should be seen shifted equatorward, although this shift is less than that upon guidance by the geomagnetic field. The maximum amplitude of the electromagnetic response of the ionosphere to the power transmission line emission decreases for an inclined geomagnetic field, but insignificantly. To date, the PLE intensity in near-Earth space has turned out to be higher than the intensity of natural radiation in this range (Schumann resonances and ion whistlers), and continues to grow with the technological development of mankind.

Keywords: power line emission, ELF radiation, geomagnetically induced currents, low-orbit satellites.

\section{INTRODUCTION}

Nowadays our planet is in the electromagnetic environment, at least in some frequency bands, which is created by industrial activity rather than by natural processes. More and more evidence is accumulating for the anthropogenic impact on natural processes in near-Earth space. This impact has been found not only in special active experiments, but also as an accidental by-product of the development of the technosphere. The anthropogenic impact is manifested in the synchronization of industrial activity with Hertz-band electromagnetic emission intensity (Pc1 pulsations) in an about $10 \%$ increase in the Pc1-pulsation activity on weekends (weekend effect) [Fraser-Smith, 1979] and in the coincidence of hour marks with the beginning of Pc1 pulsations [Zotov, Guglielmi, 2010]. These effects might have been caused by the well-known features of power consumption - its decrease on weekends, jumps at the beginning of each hour, and trigger effect on natural magnetospheric emissions.

The electromagnetic response of the ionosphere to natural and anthropogenic electromagnetic disturbances in the atmosphere (for example, lightnings) and on the ground (for example, radio transmitters) has been thoroughly studied in the very-low-frequency (VLF) band from hundreds of $\mathrm{Hz}$ to several $\mathrm{kHz}$ [Helliwell et al.,
1975; Koons et al., 1978]. Unlike VLF antennas, noticeable radiation efficiency in the extremely-low-frequency (ELF) band (of the order of the first tens to hundreds of $\mathrm{Hz}$ ) can be expected only for a very large emitter. Such artificially created large-scale emitters do exist transmission networks with $50-60 \mathrm{~Hz}$ frequencies stretched for many hundreds of kilometers. The Power Line Emission (PLE) at $50-60 \mathrm{~Hz}$ is one of the most common forms of electromagnetic radiation coming from Earth, and a manifestation of anthropogenic activity in near space.

A three-phase long-distance power transmission line should be balanced (symmetric) such that voltages and currents of each of the phases have the same amplitude, and the shift in phase is $120^{\circ}$. If at least one of these conditions is not met, the system becomes asymmetric, or unbalanced. Any imbalance leads to a decrease in the efficiency of power transmission lines and to power losses due to electromagnetic radiation [Vakhnina et al., 2018]. In most cases, a source of the imbalance is asymmetry of load (high-speed railways, induction furnaces in metallurgy, computers, lighting systems) or system damages. Considerable disruptions of operation of power systems are caused by geomagnetically induced currents (GICs) generated by rapid variations in the geomagnetic field, i.e. large values of $d B / d t$. In particular, GIC may lead to an imbalance in the mode of 
operation of power transmission lines [Vakhnina et al., 2018]. Unbalanced power transmission lines become a source of emissions at $50-60 \mathrm{~Hz}$ and their harmonics, which can propagate over long distances and even penetrate through the ionosphere. This phenomenon is qualitatively shown in Figure 1.

Although PLE events were detected in early satellite experiments in low Earth orbits (LEOs), statistics on satellite detection of them is still small. Until recently, PLE events have been observed by the satellites ISIS-1 and ISIS-2 (with a perigee/apogee 578-3526 km) [Park, Helliwell, 1981; Rodger et al., 1995], and C/NOFS (401-852 km) [Pfaff et al., 2014]. These emissions were recorded by electric antennas 73, 18.7 (ISIS-1, 2), and $20 \mathrm{~m}$ (C/NOFS) long.

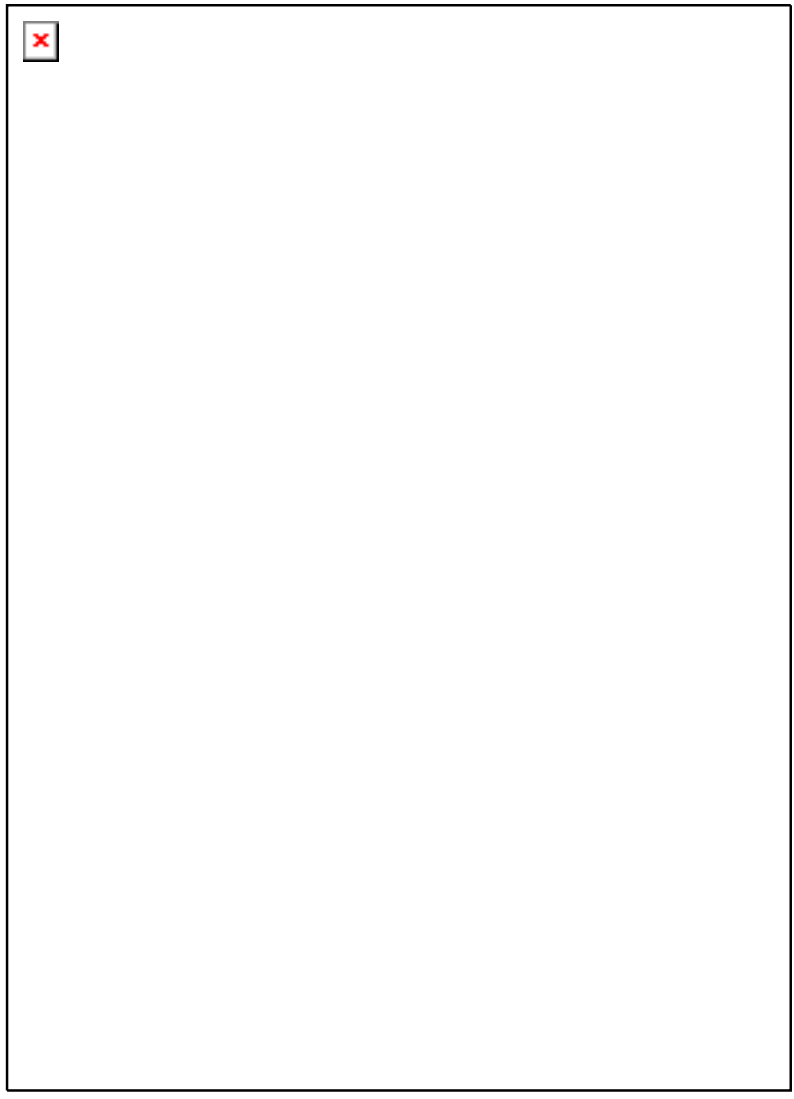

Figure 1. Illustration of PLE occurrence in the ionosphere

Due to strong non-linear elements (for example, thyristors) in consumer systems or overloading of transformers in industrial areas, $50-60 \mathrm{~Hz}$ harmonics are generated in power transmission lines. Under certain conditions, power transmission lines at high harmonics of industrial frequency can work as a very efficient traveling-wave radiating antenna [Kostrov et al., 2017]. Radiation of these harmonics in the VLF band at equidistant frequencies, spaced by $50-60 \mathrm{~Hz}$, is recorded in the ionosphere and magnetosphere as Power Line Harmonic Radiation (PLHR). PLHR is a rather weak and rare event. For example, during the DEMETER mission [Němec et al., 2006, 2007, 2008; Parrot et al., 2014], only 88 PLHR events were observed over a four-year period. This review does not cover PLHR.

Here we focus our attention on the results of PLE observations made by the Chibis-M microsatellite
[Dudkin et al., 2014, 2015; Korepanov et al., 2014, 2015] and by the DEMETER satellite [Němec et al., 2015], and also delve into approaches to modeling PLE propagation through the ionosphere to satellite heights.

\section{OBSERVATIONS OF POWER LINE EMISSIONS AT 50-60 Hz BY THE DEMETER AND CHIBIS-M SATELLITES}

PLE events in the upper ionosphere were regularly recorded by the DEMETER satellite over China [Zhang, Ma, 2018]. The power spectral density of electric field oscillations at $50 \mathrm{~Hz}$ was as high as $W_{E} \sim 0.1(\mu \mathrm{V} / \mathrm{m})^{2} / \mathrm{Hz}$, which corresponds to an electric field $E \sim \sqrt{W_{E} \Delta f} \sim 0.6 \mu \mathrm{V} / \mathrm{m}$ in the frequency band $\Delta f=4 \mathrm{~Hz}$. An example of industrial emissions recorded by DEMETER is given in Figure 2 .

A large volume of PLE observation data was obtained by the Chibis-M microsatellite in 2012-2014 [Zelenyi et al., 2014; Klimov et al., 2010]. The satellite orbit with an inclination of $52^{\circ}$ was at altitudes $350-500$ $\mathrm{km}$. In this band, emissions were recorded by an electric antenna $0.42 \mathrm{~m}$ long.

During each pass over a power transmission line in Brazil, an increase occurred in amplitude of an electric signal in a band of $60 \mathrm{~Hz}$ to $E_{f} \sim 0.6-1.0 \mu \mathrm{V} / \mathrm{m}$ and in spectral amplitude to $E_{f} \sim 0.7(\mu \mathrm{V} / \mathrm{m}) / \mathrm{Hz}^{1 / 2}$ [Dudkin et al., 2015].

The most convincing case of PLE observation on April 13, 2014 at local night time is shown in Figure 3. Chibis-M passed over Japan, where it first crossed the networks operating at a frequency of $60 \mathrm{~Hz}$, and then those working at a frequency of $50 \mathrm{~Hz}$ (top left) [Korepanov et al., 2015]. The sonogram (top right) shows the occurrence of narrow-band emission at 60 and $50 \mathrm{~Hz}$ respectively. The maximum signal at $60 \mathrm{~Hz}$ was $\sim 0.36$ $\mu \mathrm{V} / \mathrm{m}$; and at $50 \mathrm{~Hz}, \sim 0.32 \mu \mathrm{V} / \mathrm{m}$ (bottom right).

Although, in accordance with objectives of the experiment, the equipment on board the Chibis-M satellite was turned on only for short sessions over regions with expected thunderstorms, good statistics on PLE events has been collected. The sections of orbits in which PLE was detected at $50 / 60 \mathrm{~Hz}$ are summarized in Figure 4. The highest spectral amplitude up to $18(\mu \mathrm{V} / \mathrm{m}) / \mathrm{Hz}^{0.5}$ was recorded over the state of Karnataka - the Indian Silicon Valley. Moments of PLE detection mainly correspond to the location of the main transmission lines. The emissions were largely recorded in the nightside ionosphere (dark blue). The sections of orbits, where the PLE events were detected, often shifted to lower latitudes relative to power transmission lines.

In the frequency range employed (the first tens of $\mathrm{Hz}$ ), the most intense natural emissions are ion whistlers and Schumann resonances (SR). SR are eigenmodes of the Earth-lower ionosphere resonator fed by thunderstorm activity. The possibility of SR propagation into the upper ionosphere was discovered by the C/NOFS satellite [Simões et al., 2011] and confirmed by the Chibis-M satellite [Dudkin et al., 2014]. An example of simultaneous observation of industrial emission and SR on August 08, 2013 under approximately the same ionospheric 

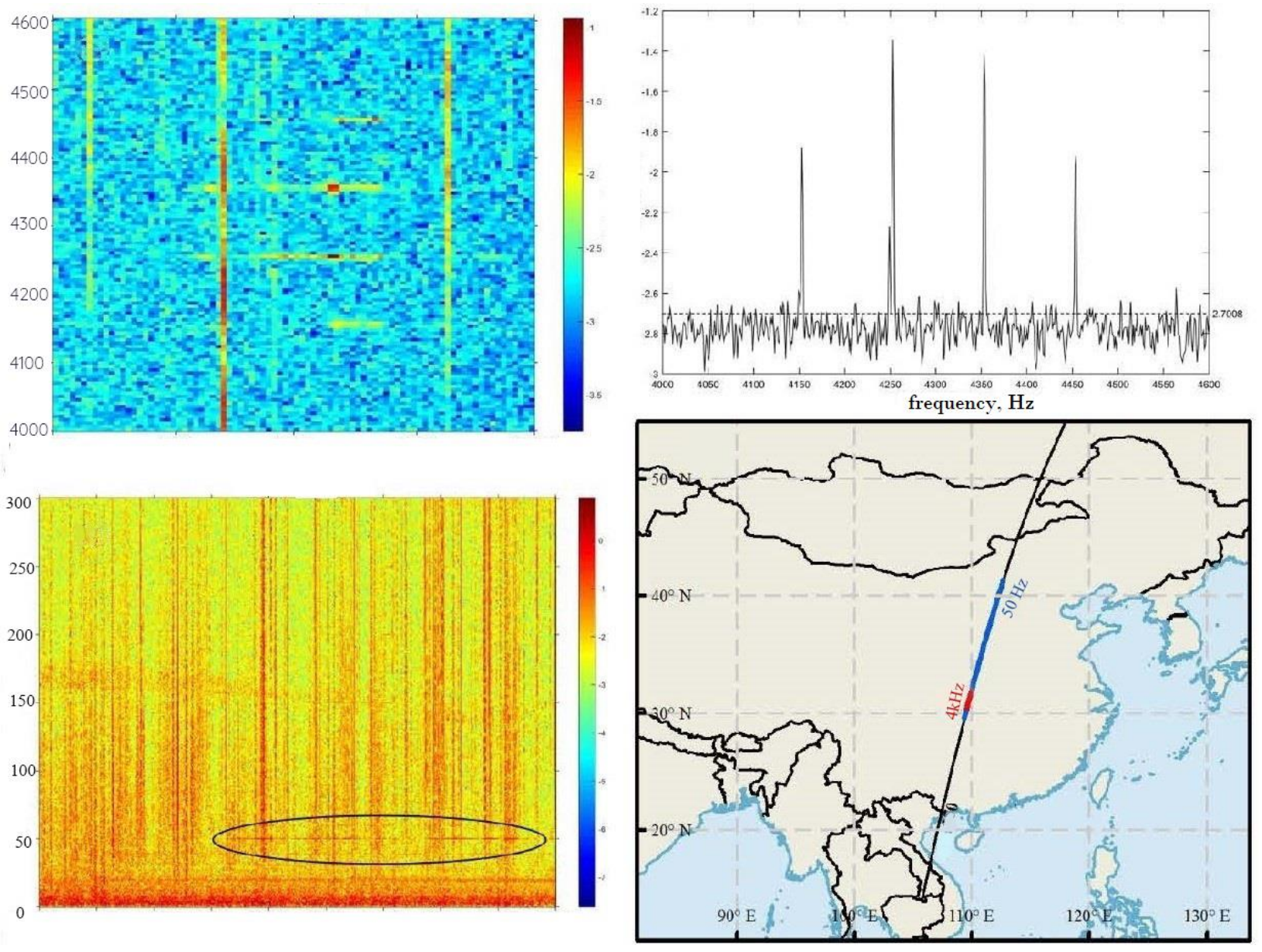

Figure 2. An example of industrial emissions recorded by the DEMETER satellite over China on November 17, 2010: The top left panel shows the PLHR sonogram frequency [Hz] - time [UT]; the top right panel, its spectrum. In the bottom left panel is the PLE sonogram frequency $[\mathrm{Hz}]$ - time [UT]; in the bottom right panel is a section of orbit, where $50 \mathrm{~Hz}$ PLE (blue) and PLHR (red) were detected (from [Zhang, Ma, 2018])
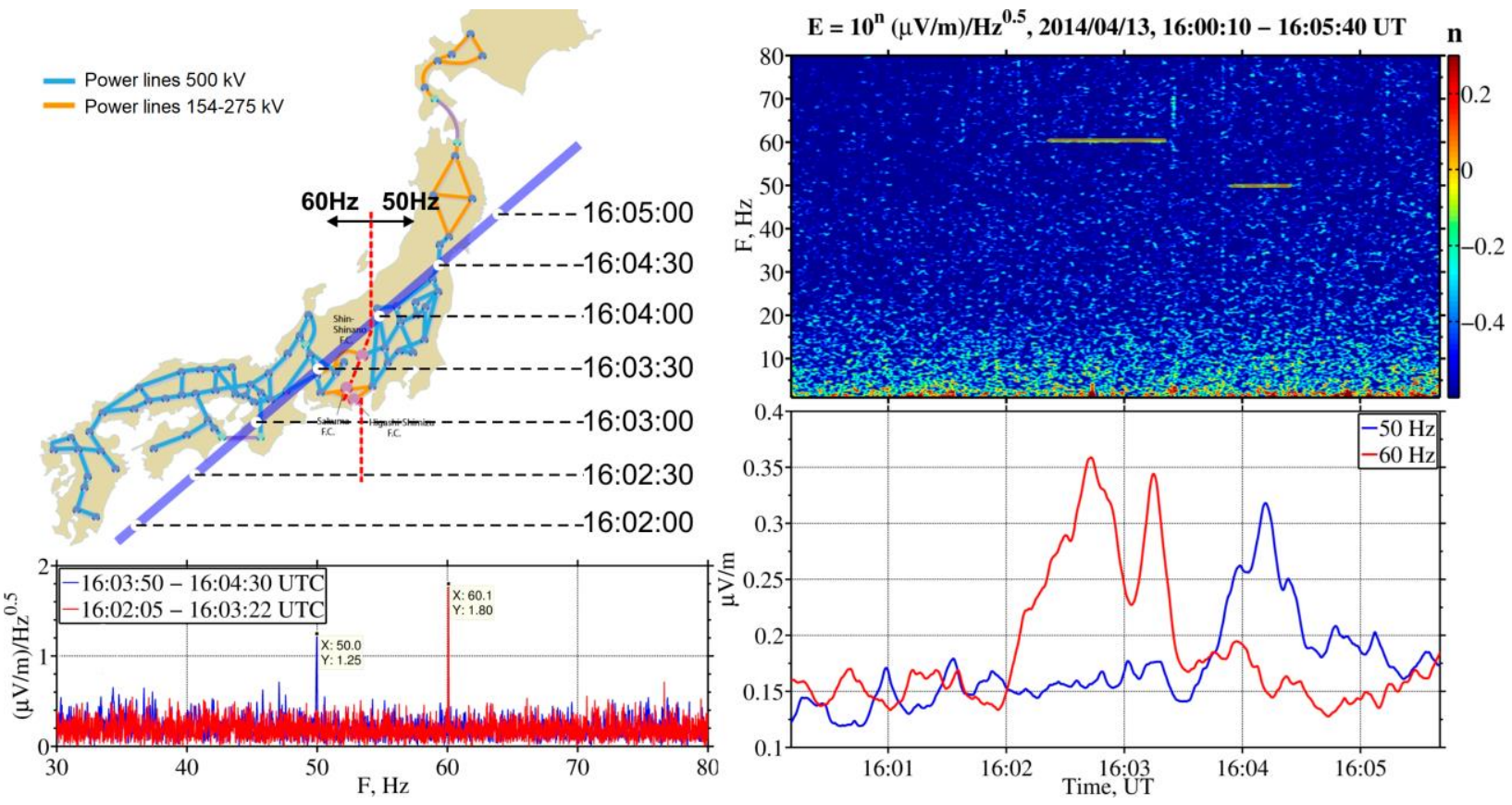

Figure 3. PLE observation by the Chibis-M microsatellite over Japan on April 13, 2014 (local night time) from [Korepanov et al., 2015]: orbital projection and configuration of trunk power systems (top left panel); sonogram of recorded emissions (top right panel); signal amplitude variations at 60 and $50 \mathrm{~Hz}$ (bottom right panel); spectrum of recorded emissions (bottom left panel) 


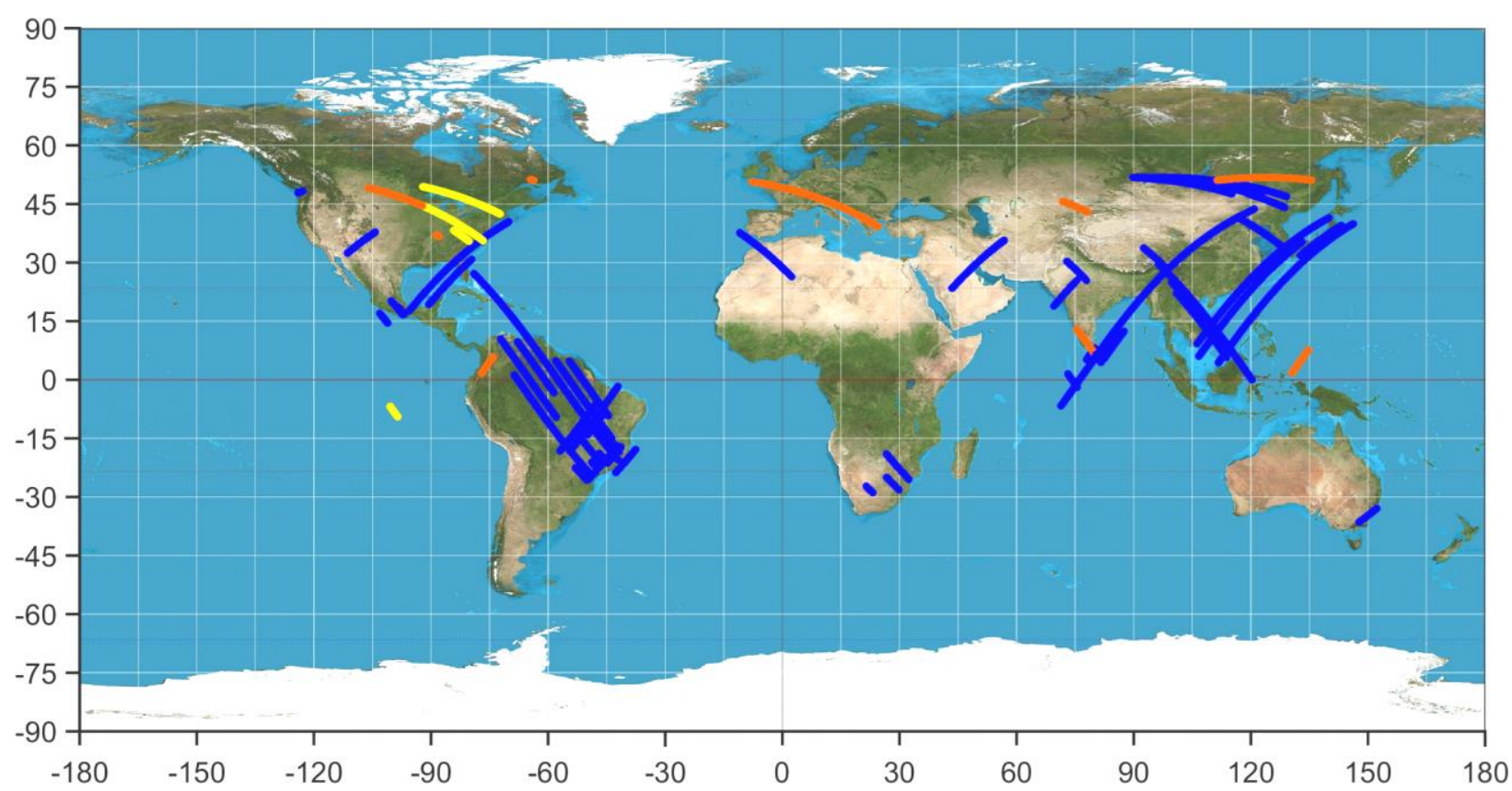

Figure 4. Sections of the Chibis-M orbits, where PLE was detected by an electric sensor at 50-60 Hz [Korepanov et al., 2015]. The orbits in the nightside ionosphere are marked with blue; the illuminated part of the trajectory, with red and yellow.

conditions is given in Figure 5. The orbit of the ChibisM satellite passed over South America, where PLE was detected, and over the Atlantic Ocean, where SR harmonics were recorded. The sonogram in Figure 5 (bottom) shows the presence of PLE at a frequency of $60 \mathrm{~Hz}$ and SR harmonics $(\sim 8,14,22 \mathrm{~Hz})$. The spectral density of the amplitude of the first three SR harmonics are, on average, $\sim 0.25, \sim 0.14$, and $\sim 0.11(\mu \mathrm{V} / \mathrm{m}) / \mathrm{Hz}^{0.5}$. By comparison, the spectral amplitude of the $60 \mathrm{~Hz}$ emission in this event was $\sim 1.2(\mu \mathrm{V} / \mathrm{m}) / \mathrm{Hz}^{0.5}$. This example suggests that in the ELF band the intensity of emissions produced by industrial activity is higher than that of such a powerful natural source as SR.

When analyzing the multi-year database of DEMETER observations, it has been noticed that the amount of radiating energy on a global scale constantly increases with an increase in the world's electricity production. A sharp increase in the number and intensity of PLE/PLHR events in space occurred in 2009, when projects on the transition to high-voltage (HV) and ultrahigh-voltage (UHV) power lines began to develop rapidly [Wu et al., 2014]. The geographic maps of the distribution of the occurrence rate of PLE at $50 \mathrm{~Hz}$ (top) and $60 \mathrm{~Hz}$ (bottom) recorded by the DEMETER satellite (Figure 6) exhibit good agreement with the world map of electric power consumption [Němec et al., 2015]. These observations demonstrate that over industrialized regions the PLE intensity is well above the average level.

Detecting PLE at a great distance from a threephase power transmission line (and even in space) indicates its unbalanced operation, to which GIC may also contribute. According to long-term DEMETER observations, the PLE intensity increases at high magnetic field variability, i.e. at high $d B / d t$ values calculated from data acquired by the ground magnetometer that was the closest to the projection of the spacecraft's orbit [Němec et al., 2015]. The PLE intensities at the

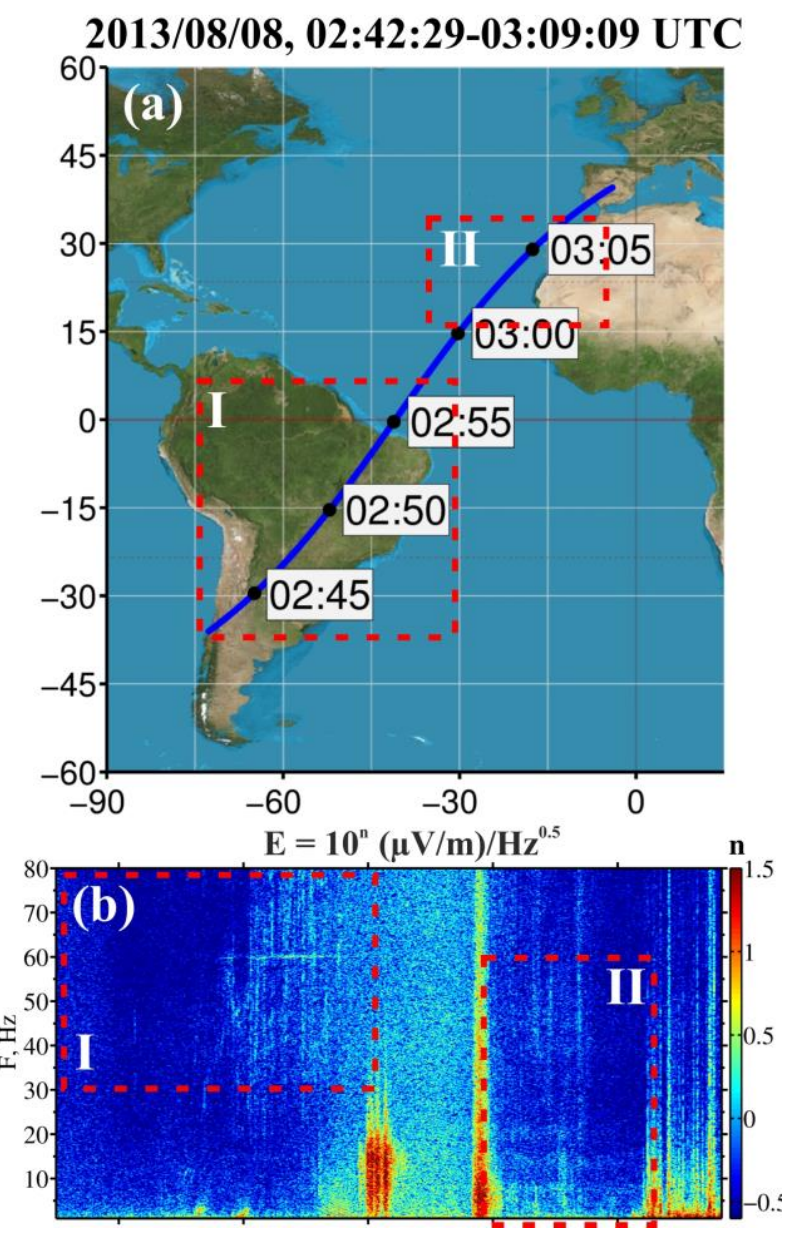

Figure 5. An example of simultaneous Chibis-M observation of industrial emission at $60 \mathrm{~Hz}$ over South America on August 08, 2013 and Schumann resonance (SR) harmonics over the Atlantic Ocean (marked with rectangular regions I and II in the top panel) [Dudkin et al., 2015]. The sonogram (bottom panel) shows the presence of PLE at $60 \mathrm{~Hz}$ and SR harmonics 

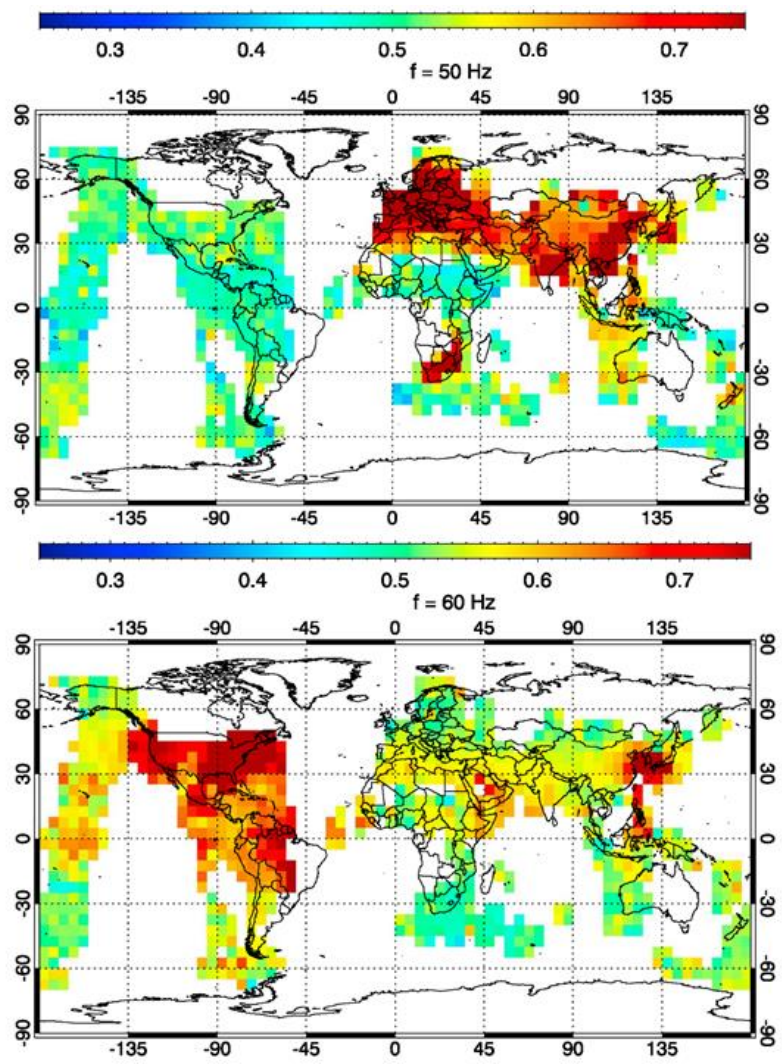

Figure 6. Geographic maps of the statistical distribution of the occurrence rate of PLE at $50 \mathrm{~Hz}$ (top) and $60 \mathrm{~Hz}$ (bottom), recorded by the DEMETER satellite [Němec et al., 2015]

fundamental frequency of the power system and at its third harmonic over Europe and North America, observed at high magnetic field variability, appear to be systematically higher than those under quiet geomagnetic conditions. The magnetic PLE component has not been detected during satellite observations yet presumably such a signal is hidden by interference and background noise.

\section{THEORETICAL MODELING OF FIELDS IN THE UPPER ATMOSPHERE \\ AND THE IONOSPHERE CREATED BY GROUND POWER TRANSMISSION LINES}

Theoretical models that make it possible to reliably describe the generation and propagation of PLE in the multilayer medium Earth - atmosphere - ionosphere have not yet been developed. Considering the ionosphere as a plasma sheet with finite conductivity turns out to be too rough. In a real situation, ELF-radiation propagation conditions change sharply depending on altitude. Moreover, PLE efficiency strongly depends on conductivity of the underlying surface. In the atmosphere $(z<70 \mathrm{~km})$, the power transmission line field is quasistatic in a medium with isotropic conductivity. In the ionosphere $(z>80 \mathrm{~km})$, the conductivity of the medium suddenly becomes anisotropic, the electromagnetic disturbance splits into two modes, and the wavelength and the phase velocity decrease sharply.
Furthermore, in the ELF band conditions arise for cyclotron resonance of a wave with ionospheric ions. It seems most correct to construct the vertical profile of ionospheric parameters with the aid of the generally accepted model IRI (International Reference Ionosphere), which provides the ionic composition, densities of charged and neutral particles, used to calculate collision frequencies and conductivity tensor components depending on altitude. Such a model of medium has been adopted in [Fedorov et al., 2016; Mazur et al., 2018], where the electromagnetic response of the ULF band to a lightning discharge in the lower atmosphere was calculated numerically. In addition, unlike radio transmitters or lightning discharges, power transmission lines up to several hundred kilometers long cannot be modeled as an infinitely small dipole, its finite scale needs to be taken into account. An approach to solving this problem is presented in [Fedorov et al., 2020], where a numerical model of the electromagnetic response of the real ionosphere to a horizontal current source in the surface layer of the atmosphere has been built. This model, however, considers the geomagnetic field as vertical so it can be used only for high latitudes. The model made it possible to quantitatively interpret the emissions detected by the DEMETER satellite at a frequency of $82 \mathrm{~Hz}$ from the system ZEVS on the Kola Peninsula [Pilipenko et al., 2019].

To analyze the effects described in the previous section, it is necessary to devise a model with an inclined geomagnetic field, which creates significant computational difficulties as the system ceases to be azimuthally symmetric. Here we present preliminary results of the new model. We consider the plane-layered vertically inhomogeneous system Earth-atmosphereionosphere whose parameters are reconstructed by the IRI model. The electromagnetic field is excited by an infinitely long east-westward power transmission line. The assumption about the infinite length of a current source seems reasonable as a first step because the height of the lower edge of the ionosphere is $\sim 80 \mathrm{~km}$, and the length of trunk power transmission lines may exceed several hundred kilometers.

The impedance boundary condition holds on Earth's surface; and the radiation condition is applied at infinity. In the plane-layered model considered, we can use the Fourier transform in horizontal coordinates. As a result, the system of Maxwell equations (after eliminating longitudinal components of field disturbance) is reduced to a system of four ordinary linear differential equations for two coupled modes propagating vertically in the inhomogeneous medium atmosphereionosphere. Coefficients of these equations and the free term, determined by the source of a disturbance, depend on the geomagnetic field inclination, the power transmission line orientation, and ionospheric plasma permittivity tensor components $\hat{\varepsilon}(z)$. The numerical calculation scheme is based on the introduction of the admittance matrix $\overline{\mathbf{Y}}$, connecting the horizontal components of electric and magnetic disturbances: $\mathbf{B}_{\tau}=\overline{\mathbf{Y}} \mathbf{E}_{\tau}$. The advantage of this numerical method is that it avoids instability of the computational process. The explicit 
form of the differential equations used in the calculations and a detailed description of the computational procedure are given in [Fedorov et al., 2020].

Amplitudes of the electric and magnetic fields in the ionosphere and atmosphere at different altitudes were numerically calculated for different ionospheric conditions and resistance of the earth's crust. The calculations were carried out for an arbitrarily chosen mid-latitude point at a geographic latitude $\Phi=44.4^{\circ} \mathrm{N}$, with an inclination $I=60.0^{\circ}$, for a frequency of $50 \mathrm{~Hz}$. The simulation results are presented for the current in a power transmission line $J_{0}=1 \mathrm{~A}$.

In the atmosphere, the excited electromagnetic field is quasistatic, but in the ionosphere - a medium with very high permittivity - the length and velocity of the ELF wave decrease sharply. In the ionosphere (above 75-80 km), the excited electromagnetic field splits into two modes. Mode (1) decays rapidly at a length of $\sim 1$ $\mathrm{km}$, while mode (2) propagates almost without decay over a scale of $\sim 10^{4} \mathrm{~km}$. In the upper ionosphere, the electromagnetic field from a ground power transmission line depends on the density of the ionosphere and resistance of the underlying layer of Earth's surface. An increase in the conductivity of the earth's crust by two orders of magnitude from $10^{-4}$ to $10^{-2} \mathrm{~S} / \mathrm{m}$ leads to a decrease in the amplitude of the excited electric field in the upper ionosphere by about an order of magnitude. Penetration through the ionosphere at night is more effective than during the day.

In a vertically inhomogeneous multicomponent plasma there are several characteristic points affecting propagation and attenuation of ELF waves:

- the height at which the cyclotron resonance with ions of a given type takes place: $\omega=\Omega_{\mathrm{i}}(\mathrm{z})$;

- the reflecting point $z=z_{\mathrm{R}}$ that separates the transparent region, where the refractive index $n^{2}>0$, from the stop region (evanescent propagation), where $n^{2}<0$;

- the region of crossover of two modes at a height $z=z^{*}$, where off-diagonal tensor elements $\operatorname{Re} g=0$, and tensor eigenvalues coincide $\varepsilon_{1}=\varepsilon_{2}$. For the finite horizontal wavenumber $k_{1}=0.01 \mathrm{~km}^{-1}$, when approaching the cross point $z^{*}=1040 \mathrm{~km}$, the longitudinal wavenumbers $k_{3}^{(1)}$ and $k_{3}{ }^{(2)}$ come close together, but a gap of finite width remains between the branches.

Vertical profiles of the squared refractive index of the two modes $n_{1,2}^{2}$ are shown in Figure 7. Mode (1) has a reflecting point at $z_{\mathrm{R}}=980 \mathrm{~km}$. Below $z_{\mathrm{R}}$, mode (1) falls into the stop region, and only above this point it becomes propagating. For mode (2), the entire ionosphere proves to be a transparent region. The vertical profile of gyrofrequencies indicates that the cyclotron resonance is possible with $\mathrm{N}^{+}$ions at an altitude of $250 \mathrm{~km}$, and with $\mathrm{He}^{+}$ions at $z \sim 600 \mathrm{~km}$. Since ion densities at these altitudes are low, these regions do not have a significant effect on wave propagation.

In a right-handed $(\mathrm{RH})$ polarized wave, the magnetic and electrical components $E$ and $B$ rotate clockwise when viewed along the direction of wave propagation; and in a left-handed ( $\mathrm{LH})$ polarized wave, counterclockwise.

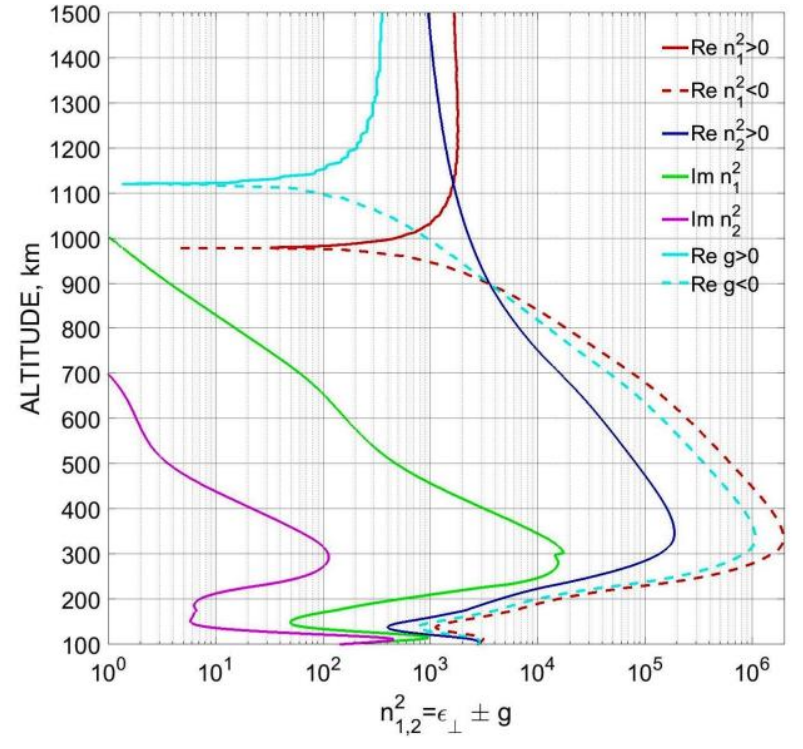

Figure 7. Vertical profiles of the squared refractive index of two modes $n_{1.2}^{2}$ and the element $g$ of the dielectric permeability tensor ( $50 \mathrm{~Hz}$ frequency). The reflection point of mode (1) is labeled $z_{R}$. To make the plot more compact, the dependences $\operatorname{Re} n^{2}(z)$ are shown by solid lines; and $\operatorname{Im} n^{2}(z)$, by dashed lines of the same color.

For quasi-longitudinal propagation, polarization of both modes is almost circular, and the sense of wave field rotation (sense of ellipticity) is determined by the sense of the off-diagonal tensor element $g$ because

$$
\left(E_{2} / E_{1}\right) \sin I ; \operatorname{mi} \operatorname{Re}(g /|g|) .
$$

Mode (1) has RH polarization; mode (2) has LH polarization. At the cross point $\left(z=z^{*} \sim 1040 \mathrm{~km}\right)$, where $\mathrm{Reg} \rightarrow 0$, polarization of both modes becomes linear, while the electric field of mode (1) is directed along the parallel; and that of mode (2), along the meridian.

The complex wave pattern of ELF wave propagation in the ionosphere defies analytical consideration and requires numerical simulation. To identify the influence of the geomagnetic field inclination, we have calculated the horizontal structure of the electric field disturbance at an altitude of $600 \mathrm{~km}$ for different inclinations $I$, but for the same ionospheric plasma model (Figure 8). At this height, the maximum values of $\sim 0.2 \mu \mathrm{V} / \mathrm{m}$ are seen for the vertical field $\mathbf{B}_{0}\left(I=90^{\circ}\right)$ and slowly decrease with a decrease in $I$ to $\sim 0.15 \mu \mathrm{V} / \mathrm{m}$ at $I=60^{\circ}$. Since the ELF disturbance in the ionosphere is not channeled by the geomagnetic field as an Alfvén wave in the MHD approximation, the maximum response in the ionosphere should slightly shift from the field line adjacent to the source. This effect can be seen in Figure 8, which shows that when propagating upward the maximum of the disturbance deviates from the geomagnetic field line. For example, at the altitude of the DEMETER satellite $(z=660 \mathrm{~km})$, the maximum amplitude of the electrical component $\max \left|E_{y}\right|$ is displaced from the vertical by $\Delta x \sim 250 \mathrm{~km}$, whereas projecting the disturbance along 


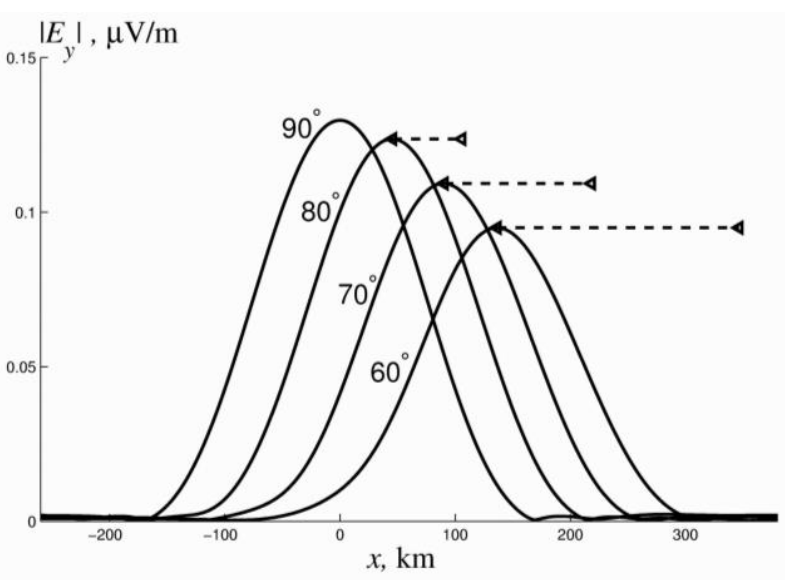

Figure 8. Horizontal structure of the electric field disturbance $\left|E_{y}(x)\right|$ at an altitude of $600 \mathrm{~km}$ for different inclinations $I$, but for the same ionospheric plasma model. The position of the source on the ground corresponds to $x=0$. Open and shaded triangles represent the displacement along the field line and the actual position of the maximum signal intensity in the ionosphere. The distance between them (horizontal dashed lines) shows deviation of the signal from guided propagation along the field line

an oblique magnetic field with $I=60^{\circ}$ would give a displacement $\Delta x=z \operatorname{ctg} I \sim 380 \mathrm{~km}$.

The maximum electrical component $\max |E|(z)$, where $E=\sqrt{E_{x}^{2}+E_{y}^{2}}$, observed at different altitudes at night, is displayed in Figure 9. Due to the refractive index profile nonmonotonic variations in altitude, this value varies between 0.07 and $0.17 \mu \mathrm{V} / \mathrm{m}$.

\section{COMPARING SIMULATION RESULTS WITH SATELLITE OBSERVATIONS ON LEO SPACECRAFT}

When the Chibis-M microsatellite $(500 \mathrm{~km})$ passed over high-voltage power transmission lines, an increase in the electric signal amplitude averaged $E \sim 0.6-1.0$ $\mu \mathrm{V} / \mathrm{m}$; and in the spectral amplitude density, $E_{f} \sim 0.7$ $(\mu \mathrm{V} / \mathrm{m}) / \mathrm{Hz}^{0.5}$ [Dudkin et al., 2014, 2015; Korepanov et al., 2014].

Over 6.5 years of operation, the DEMETER satellite $(660 \mathrm{~km})$ has detected many PLE events at the fundamental frequency of $50 \mathrm{~Hz}$ of the local power system over China [Zhang, Ma, 2018]. The spectral power density of electric field fluctuations was as high as $W_{E} \sim 0.1$ $(\mu \mathrm{V} / \mathrm{m})^{2} / \mathrm{Hz}$, which corresponds to the electric field with an amplitude $E \sim 0.6 \mu \mathrm{V} / \mathrm{m}$ in a $4 \mathrm{~Hz}$ band. In an extensive study based on DEMETER data, PLE power spectral densities over Europe and the United States range between $W_{E} \sim 0.5-2.0(\mu \mathrm{V} / \mathrm{m})^{2} / \mathrm{Hz}$ [Němec et al., 2008]. The average PLE spectral amplitude recorded by the electric antenna of the low-latitude satellite C/NOFS $(400-850 \mathrm{~km})$ varied around $E_{f} \sim 0.25-0.5(\mu \mathrm{V} / \mathrm{m}) / \mathrm{Hz}^{0.5}$ [Pfaff et al., 2014]. The electric field amplitude $E \sim 0.5-$ $1.0 \mu \mathrm{V} / \mathrm{m}$, which roughly corresponds to the amplitudes observed by the Chibis-M and DEMETER satellites.

According to the simulation results, PLE with an intensity of $\sim 1 \mu \mathrm{V} / \mathrm{m}$ observed at midlatitudes can be

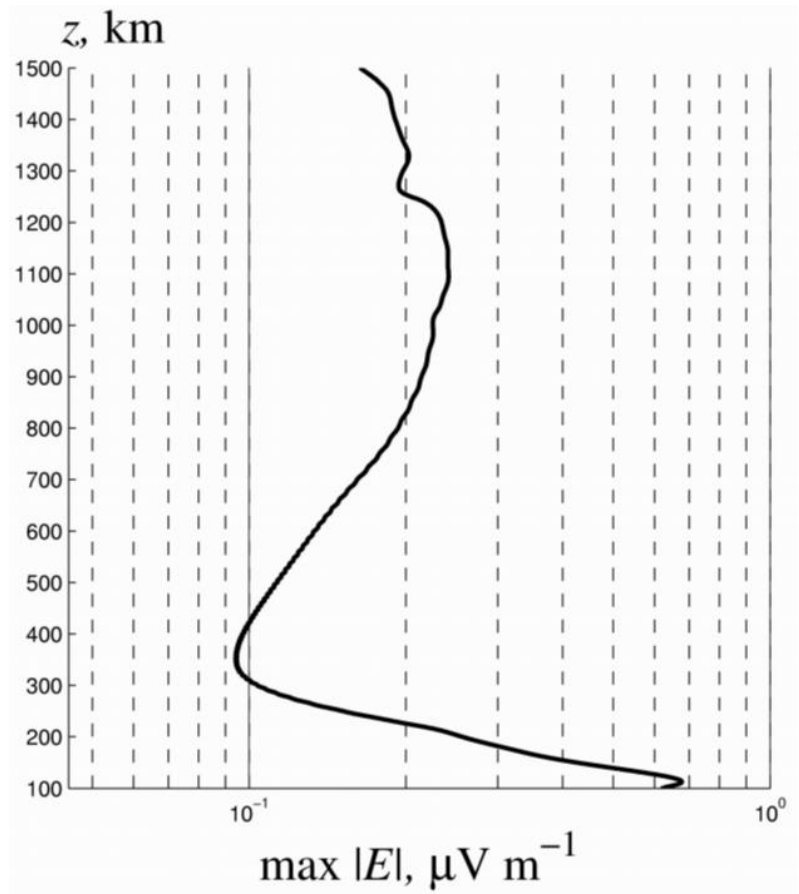

Figure 9. Maximum value of the electrical component of radiation $\max |E|(x, z)$ at various altitudes at night

excited by an unbalanced current in a power transmission line $J_{0} \sim 8 \div 10$ A over the earth's crust with a conductivity of $10^{-3} \mathrm{~S} / \mathrm{m}$. The estimated value of the unbalanced current is small compared to the typical currents transmitted in power lines (of the order of hundreds to thousands of A).

It has been noted that the PLE intensity is usually higher at night than during the day [Němec et al., 2015]. The numerical simulation carried out in [Fedorov et al., 2020] for vertical $\mathbf{B}_{0}$ has shown that this asymmetry in day and night is caused by a large attenuation of waves during propagation in the dayside ionosphere. Obviously, this asymmetry will also remain for oblique $\mathbf{B}_{0}$.

At middle and low latitudes with an inclined geomagnetic field, the maximum response in the upper ionosphere to the power transmission line radiation should be seen shifted equatorward. This shift is less than that caused by the guiding of electromagnetic disturbances by the background magnetic field. For example, for the $50 \mathrm{~Hz}$ radiation, the shift of the radiation maximum should be $\sim 250 \mathrm{~km}$ at the altitude of the DEMETER orbit $(660 \mathrm{~km})$. This shift of PLE to lower latitudes relative to a power transmission line was in fact observed by the Chibis-M satellite (see Figure 4).

The model presented takes into account the ionospheric structure in the most realistic way, but it needs further elaboration. In particular, it would be desirable to consider the finite length of a transmission line. This will most likely somewhat lower the estimate of the ionospheric response to the power transmission line operation.

\section{CONCLUSION: POTENTIAL OF FURTHER RESEARCH}

Industrial electromagnetic activity in the ELF band (50-60 $\mathrm{Hz}$ and harmonics) can be monitored using 
LEO microsatellites [Rothkaeh, Parrot, 2005]. The PLE intensity in NES gradually increases with time, it has increased significantly after the introduction of high-voltage transmission lines into power industry [Wu et al., 2019]. Simulation shows that unbalanced currents with an intensity of $\sim 10 \mathrm{~A}$ in power transmission lines excite PLE events with an amplitude of $\sim 1$ $\mu \mathrm{V} / \mathrm{m}$, which is sufficient to detect by electric sensors on board LEO satellites in the nightside upper ionosphere. Electromagnetic pollution of near-Earth space by ground power transmission lines will constantly increase with the industrial development of society.

We are grateful to Korepanov V.A. for many years of cooperation on the problems addressed in this work.

The work was performed under State assignments of IPE and IKI.

\section{REFERENCES}

Dudkin D., Pilipenko V., Korepanov V., Klimov S., Holzworth R. Electric field signatures of the IAR and Schumann resonance in the upper ionosphere detected by Chibis-M microsatellite. J. Atmos. Solar-Terr. Phys. 2014,. vol. 117, pp. 81-87.

Dudkin F., Korepanov V., Dudkin D., Pilipenko V., Pronenko V., Klimov S. Electric field of the power terrestrial sources observed by microsatellite Chibis-M in the Earth's ionosphere in frequency range 1-60 Hz. Geophys. Res. Lett. 2015, vol. 42, pp. 5686-5693.

Fedorov E., Mazur N., Pilipenko V., Baddeley L. Modeling the high-latitude ground response to the excitation of the ionospheric MHD modes by atmospheric electric discharge. J. Geophys. Res. 2016, vol. 121, pp. 11282-11301. DOI: 10.1002/2016JA023354.

Fedorov E., Mazur N., Pilipenko V., Vakhnina V. Modeling ELF electromagnetic field in the upper ionosphere from power transmission lines. Radio Sci. 2020, vol. 121, 55, e2019RS006943. DOI: 10.1029/2019RS006943.

Fraser-Smith A. A weekend increase in geomagnetic activity. J. Geophys. Res. 1979, vol. 84, pp. 2089-2096. DOI: 10.1029/JA084iA05p02089.

Helliwell R.A., Katsufrakis J.P., Bell T.F., Raghuram R. VLF line radiation in the Earth's magnetosphere and its association with power system radiation. J. Geophys. Res. 1975, vol. 80, pp. 4249-4258.

Klimov S., Korepanov V., Marusenkov A., Novikov D. The study of electromagnetic parameters of space weather, micro-satellite "Chibis-M", in: Sandau, R., H.-P. Roeser, A Valenzuela (Eds.), Small satellite missions for earth observation: New developments and trends, Springer-Verlag Berlin Heidelberg, 2010, pp. 95-102. DOI: 10.1007/978-3-64203501-2.

Koons H.C., Dazey M.H., Edgar B.C. Satellite observation of discrete VLF line radiation within transmitter-induced amplification bands. J. Geophys. Res. 1978, vol. 83, pp. 38873889.

Korepanov V., Dudkin D., Dudkin F. Study of electromagnetic processes in the ionosphere onboard microsatellite Chibis-M, In: Fedorov O. (ed.), Space research in Ukraine. Akademperiodyka Kyiv, 2014, pp. 7-12.

Korepanov V.E., Dudkin F.L., Pronenko V.A. Observations of radiation from power lines in near-Earth space. Geomagnetism and Aeronomy. 2015, vol. 55, pp. 706-711. DOI: $10.1134 / \mathrm{S} 0016793215050084$.

Kostrov A.V., Gushchin M.E., Strikovsky A.V. Generation and emission of harmonics of power lines. Geomagnetism and Aeronomy. 2017, vol. 57, pp. 482-490.
DOI: 10.1134/S0016793217030094.

Mazur N.G., Fedorov E.N., Pilipenko V.A., Vakhnina V. ULF electromagnetic field in the upper ionosphere excited by lightning. J. Geophys. Res. 2018, vol. 123, pp. 6692-6702. DOI: $10.1029 / 2018 J A 025622$.

Němec F., Santolík O., Parrot M., Berthelier J. Power line harmonic radiation (PLHR) observed by the DEMETER spacecraft. J. Geophys. Res. 2006, vol. 111, A04308. DOI: 10.1029/2005JA011480.

Němec F., Santolík O., Parrot M., Berthelier J. Power line harmonic radiation: A systematic study using DEMETER spacecraft. Adv. Space Res. 2007, vol. 40, pp. 398-403. DOI: 10.1016/ j.asr.2007.01.074.

Němec F., Santolík O., Parrot M., Bortnik J. Power line harmonic radiation observed by satellite: Properties and propagation through the ionosphere. J. Geophys. Res. 2008, vol. 113, A08317. DOI: 10.1029/2008JA013184.

Němec F, Parrot M, Santolik O. Power line harmonic radiation observed by the DEMETER spacecraft at $50 / 60 \mathrm{~Hz}$ and low harmonics. J. Geophys. Res. 2015, vol. 120, pp. 8958967.

Park C.G., Helliwell R.A. Power line radiation in the magnetosphere, Adv. Space Res. 1981, vol. 1, pp. 423-437.

Parrot M., Němec F., Santolík O. Statistical analysis of VLF radio emissions triggered by power line harmonic radiation and observed by the low-altitude satellite DEMETER. $J$. Geophys. Res. 2014, vol. 119. DOI: 10.1002/2014JA020139.

Pfaff R., Freudenreich H., Simões F., Liebrecht, M.C., Farrell W. Observations of $50 / 60 \mathrm{~Hz}$ power line radiation in the low latitude ionosphere detected by the electric field instrument on the C/NOFS satellite, General Assembly and Scientific Symposium, XXXI ${ }^{\text {th }}$ URSI, Beijing, China, 2014, Book of abstracts, IEEE. DOI: 10.1109/URSIGASS.2014. 6929584.

Pilipenko V.A., Parrot M., Fedorov E.N., Mazur N.G. Electromagnetic field in the upper ionosphere from ELF ground-based transmitter. J. Geophys. Res. 2019, vol. 124. DOI: 10.1029/2019JA026929.

Rothkaehl H., Parrot M. Electromagnetic emissions detected in the topside ionosphere related to the human activi-J. Atmos. Solar-Terr. Phys. 2005, vol. 67, pp. 821-828.

Rodger C.J., N.R. Thomson, R.L. Dowden VLF line radıation observed by satellite. J. Geophys. Res. 1995, vol. 100, pp. 5681-5689. DOI: 10.1029/94JA02865.

Simões F.A., Pfaff R.F., Freudenreich H.T. Satellite observations of Schumann resonances in the Earth's ionosphere. Geophys. Res. Lett. 2011, vol. 38, L22101. DOI: 10.1029/ 2011GL049668.

Vakhnina V.V., Kuvshinov A.A., Shapovalov V.A., et al. Mechanisms of the impact of quasi-DC geomagnetically induced currents on electrical networks, M., Infra-Engineering, 2018, 256 p.

Wu J., Fu J.J., Zhang C. Propagation characteristics of power line harmonic radiation in the ionosphere, Chinese Physics B. 2014, vol. 23, pp. 034102-034107. DOI: 10.1088/ 1674-1056/23/3/034102.

Wu J., Guo Q., Yue C., Li X. Special electromagnetic interference in the ionosphere directly correlated with power system. IEEE Transactions on Electromagnetic Compatibility. 2019. DOI: 10.1109/TEMC.2019.2918280.

Zelenyi L.M., Gurevich A.V., Klimov S.I., Angarov V.N., Batanov O.V., Bogomolov A.V., et al. The academic microsatellite Chibis-M, Kosmicheskie Issledovanija [Cosmic Res.]. 2014, vol. 52, no. 2, pp. 93-105.

Zhang C., Ma Q. Influences of radiation from terrestrial power sources on the ionosphere above China based on satellite observation, 2nd International Workshop on Renewable Energy and Development, Conf. Series: Earth and Environmental Science. 2018, vol. 153, 042002. DOI: 10.1088/1755- 
1315/153/4/042002.

Zotov O.D., Guglielmi A.A. Problems of synchronism of electromagnetic and seismic events in the dynamic system magnetosphere-technosphere-lithosphere. Solar-Terr. Phys. 2010, vol. 16, pp. 19-25.

This paper is based on material presented at the 16th Annual Conference on Plasma Physics in the Solar System, February 8 12, 2021, IKI RAS.

How to cite this article

Pilipenko V.A., Fedorov E.N., Mazur N.G., Klimov S.I. Electromagnetic pollution of near-Earth space by power line emission. Solar Terrestrial Physics. 2021. Vol. 7. Iss. 3. P. 105-113. DOI: $10.12737 /$ stp-73202107. 